\title{
Evaluation of optimized magnetic resonance perfusion imaging scanning time window after contrast agent injection for differentiating benign and malignant breast lesions
}

\author{
JIE DONG ${ }^{1}$, DAWEI WANG ${ }^{1}$, ZHENSHEN MA $^{1}$, GUODONG DENG $^{2}$, LANHUA WANG $^{3}$ and JIANDONG ZHANG ${ }^{2}$ \\ Departments of ${ }^{1}$ Radiology and ${ }^{2}$ Radiation Oncology, Qianfoshan Hospital \\ Affiliated to Shandong University, Jinan, Shandong 250014; ${ }^{3}$ Department of Oncology, \\ Linqing People's Hospital, Linqing, Shandong 252600, P.R. China
}

Received July 21, 2015; Accepted December 19, 2016

DOI: $10.3892 /$ etm.2017.4060

\begin{abstract}
The aim of the study was evaluate the 3.0 T magnetic resonance (MR) perfusion imaging scanning time window following contrast injection for differentiating benign and malignant breast lesions and to determine the optimum scanning time window for increased scanner usage efficiency and reduced diagnostic adverse risk factors. A total of 52 women with breast abnormalities were selected for conventional MR imaging and T1 dynamic-enhanced imaging. Quantitative parameters [volume transfer constant $\left(\mathrm{K}_{\text {trans }}\right)$, rate constant $\left(\mathrm{K}_{\mathrm{ep}}\right)$ and extravascular extracellular volume fraction $\left.\left(\mathrm{V}_{\mathrm{e}}\right)\right]$ were calculated at phases 10, 20, 30, 40 and 50, which represented time windows at 5, 10, 15, 20 and $25 \mathrm{~min}$, respectively, following injection of contrast agent. The association of the parameters at different phases with benign and malignant tumor diagnosis was analyzed. MR perfusion imaging was verified as an effective modality in the diagnosis of breast malignancies and the best scanning time window was identified: i) Values of $K_{\text {trans }}$ and $K_{e p}$ at all phases were statistically significant in differentiating benign and malignant tumors $(\mathrm{P}<0.05)$, while the value of $\mathrm{V}_{\mathrm{e}}$ had statistical significance only at stage 10, but not at any other stages $(\mathrm{P}>0.05)$; ii) values of $\mathrm{V}_{\mathrm{e}}$ in benign tumors increased with phase number, but achieved no obvious changes at different phases in malignant tumors; iii) the optimum scanning time window of breast perfusion imaging with 3.0 T MR was between phases 10 and 30 (i.e., between 5 and $15 \mathrm{~min}$ after contrast agent injection). The variation trend of $\mathrm{V}_{\mathrm{e}}$ values at different phases may serve as a diagnostic reference for differentiating benign and malignant breast abnormalities. The most efficient scanning time window
\end{abstract}

Correspondence to: Professor Jiandong Zhang, Department of Radiation Oncology, Qianfoshan Hospital Affiliated to Shandong University, 16766 Jingshi Road, Jinan, Shandong 250014, P.R. China E-mail: zhangjd165@sina.com

Key words: breast lesions, malignant and benign, magnetic resonance imaging, perfusion imaging, scanning time window was indicated to be 5 min after contrast injection, based on the observation that the $\mathrm{V}_{\mathrm{e}}$ value only had statistical significance in diagnosis at stage 10 . However, the optimal scanning time window is from 5 to 15 min following the injection of contrast agent, since that the variation trend of $V_{e}$ is able to serve as a diagnostic reference.

\section{Introduction}

Breast cancer is one of the most common malignant tumors endangering the health of women. Molybdenum-target mammography is the main screening method for breast cancer; however, the sensitivity of this method is only reported to be $69-90 \%$, and the sensitivity in dense breast is decreased further to $48 \%(1,2)$. As magnetic resonance imaging (MRI) is widely adopted in clinical use, dynamic contrast enhancement MRI (DCE-MRI) has been applied for monitoring tumor angiogenesis and hemodynamic changes in order to provide valuable information for the diagnosis of lesions and the selection of treatment options. The meta-analysis conducted by Peters et al (3), to evaluate the diagnostic efficacy of MRI for breast cancer, showed that the sensitivity and specificity were 90 and $72 \%$, respectively. Numerous studies have been performed to evaluate magnetic resonance perfusion imaging in breast lesion diagnosis in recent years, but few studies have evaluated the optimized scanning window following contrast agent injection (4-6).

Although MRI is recognized as a safer diagnostic modality compared with X-ray-based methods, certain biological effects such as perfusion and metabolism, and physical effects such as heating and motion may have compound adverse effects on diagnostic results $(7,8)$. The determination of the optimum scanning window following contrast injection is of particular interest, as it should improve breast perfusion scanning efficiency and reduce adverse imaging effects, on the premise of utilizing the best quality images.

Therefore, the present study evaluated the image quality and diagnostic specificity of magnetic resonance perfusion images from different scanning windows following contrast injection and identified the most efficient and effective scanning window for breast lesions. 


\section{Materials and methods}

Patients. The study population comprised 52 female patients undergoing breast MRI dynamic enhanced scanning after they were found to have breast lumps using ultrasound examination and molybdenum target X-ray mammography. The ages of the patients ranged from 23 to 68 years. Patients included in this study had no MRI contraindications, had not received any chemotherapy and did not show renal insufficiency problems. Informed consent was obtained for all patients prior to examination. All patients with lesions were confirmed by pathology, including 36 cases of malignant disease (69.2\%): 32 cases of infiltrating ductal carcinoma, 2 cases of intraductal carcinoma, 1 case of inflammatory breast cancer, 1 case of lipid-rich invasive carcinoma; and 16 cases of benign tumors (30.8\%): 7 cases of fibroadenoma, 6 cases of mammary gland hyperplasia, 1 case of intraductal papilloma, 1 case of borderline phyllodes tumor and 1 case of lipoma. This research was approved by the local ethics committee of Qianfoshan Hospital (Jinan, China), and all patients provided written informed consent.

Instruments and imaging methods. A Siemens Magnetom Skyra 3.0 T MRI scanner (Siemens AG, Munich, Germany) with a dedicated bilateral eight-channel phased array breast coil was used. Patients were scanned in prone position with bilateral breasts naturally hanging in the coil. Following regular transverse, sagittal and coronary positioning with a localizer sequence, a dual flip-angle ( 3 and $16^{\circ}$ ) T1-weighted sequence was applied [repetition time (TR)/echo time (TE), $7.84 \mathrm{msec} / 7.84 \mathrm{msec}$; field of view (FOV), $340 \mathrm{~mm}$; matrix, 224x224; slice thickness, $1.5 \mathrm{~mm}$; slice distance, $0.3 \mathrm{~mm}$; one excitation). For dynamic contrast-enhanced T1 perfusion imaging, a series of single flip angle $\left(10^{\circ}\right)$ T1-weighted sequences were applied (TR/TE $5.61 \mathrm{msec} / 1.74 \mathrm{msec}$; FOV, $340 \mathrm{~mm}$; matrix, 224x224; slice thickness, $1.5 \mathrm{~mm}$; slice distance, $0.3 \mathrm{~mm}$; one excitation for each phase acquisition; single phase scanning time, $30 \mathrm{sec}$; total scanning time, $26 \mathrm{~min})$. Patients were injected with $20 \mathrm{ml}$ intravenous high contrast agent gadobenate dimeglumine (Bracco Imaging S.p.A.,Milan, Italy) after the second single phase scan, followed by $20 \mathrm{ml}$ saline water injection at a flow rate of $5 \mathrm{ml} / \mathrm{sec}$. Fifty continuous phase images were collected thereafter.

Image processing and evaluation. All data were transferred to a SYGNO VE40A workstation (Siemens AG) for post-processing using TISSUE 4D software. Arterial input functions (AIFs) at different times were acquired in obvious mammary gland artery or thoracic artery, selected manually in the images at phases of 10 (5 min after injection of contrast agents), 20 (10 $\mathrm{min}), 30(15 \mathrm{~min}), 40(\mathrm{~min}) 20$ and 50 (25 min). Certain regions of interest (ROI), displayed by pseudo-color maps, were selected to measure the change of the following quantitative parameters in different pathology periods: i) Volume transfer constant $\left(\mathrm{K}_{\text {trans }}\right)$, describing the diffusion rate of the contrast agent from intravascular space to extravascular space; ii) the rate constant $\left(\mathrm{K}_{\mathrm{ep}}\right)$, referring to the diffusion rate of the contrast agent from intracellular space to intravascular space; iii) extravascular extracellular volume fraction $\left(\mathrm{V}_{\mathrm{e}}\right)$, indicating the ratio of the contrast agent permeated from extracellular space outside the vascular volume to

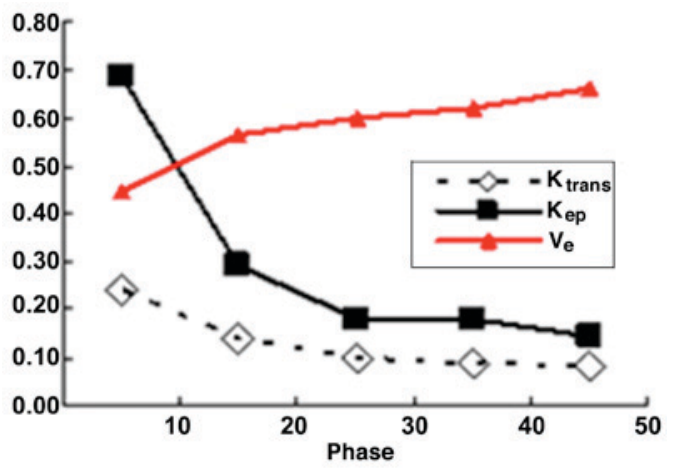

Figure 1. Indicator changes for benign breast lesions. $\mathrm{K}_{\text {trans }}$, volume transfer constant; $\mathrm{K}_{\mathrm{ep}}$, rate constant; $\mathrm{V}_{\mathrm{e}}$, extravascular extracellular volume fraction.

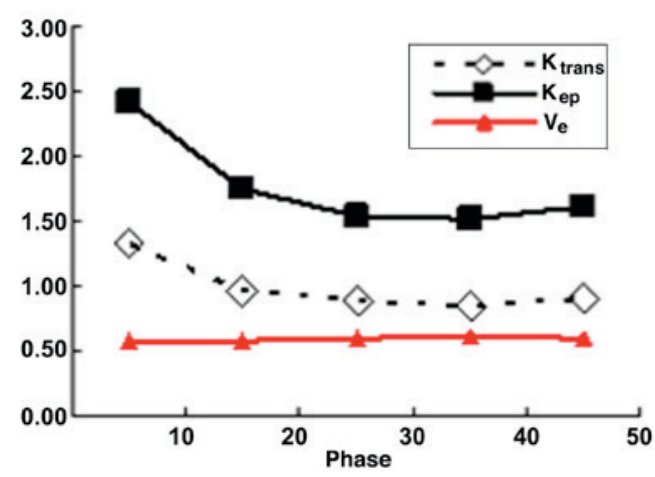

Figure 2. Indicator changes for malignant breast lesions. $\mathrm{K}_{\text {trans }}$, volume transfer constant; $\mathrm{K}_{\mathrm{ep}}$, rate constant; $\mathrm{V}_{\mathrm{e}}$, extravascular extracellular volume fraction.

the volume of the ROI. The criteria of ROI selection were regions without necrotic tissue, hollow space, calcification and blood vessels. Quantitative parameters for pathological identification were averaged over three consecutive slices containing the largest cross section of the selected abnormal lump zone.

Statistical analysis. SPSS software (version 17.0; SPSS, Inc., Chicago, IL, USA) was used for the study. Data are presented in the form of the mean \pm standard deviation. Quantitative comparisons between benign and malignant tumor groups, and between various phases, were achieved using the t-test. $\mathrm{P}<0.05$ was considered to indicate a statistically significant difference.

\section{Results}

Quantitative comparisons between benign and malignant tumor. As shown in Tables I and II, the $\mathrm{K}_{\text {trans }}$ and $\mathrm{K}_{\mathrm{ep}}$ values at all phases had statistical significance in the differentiation of benign and malignant tumors $(\mathrm{P}<0.05)$. For $\mathrm{V}_{\mathrm{e}}$ values, no statistical significance was observed in the differentiation of benign and malignant tumors at all phases, with the exception of phase 10. However, $\mathrm{V}_{\mathrm{e}}$ values increased as the scanning delay time was prolonged in benign lesions, while they exhibited no significant changes at different scanning phases in malignant lesions. Detailed variation tendencies are shown in Figs. 1 and 2. Moreover, typical images differentiating benign and malignant breast lesions are presented in Figs. 3 and 4, respectively. 
Table I. Indicator values in benign and malignant lesions (mean \pm standard deviation).

\begin{tabular}{lcccccc}
\hline \multirow{2}{*}{ Lesions } & & \multicolumn{5}{c}{ Phase } \\
\cline { 3 - 7 } & Indicators & 10 & 20 & 30 & 40 & 50 \\
\hline \multirow{2}{*}{ Benign } & $\mathrm{K}_{\text {trans }}$ & $0.242 \pm 0.182$ & $0.136 \pm 0.088$ & $0.100 \pm 0.062$ & $0.088 \pm 0.050$ & $0.083 \pm 0.048$ \\
& $\mathrm{~K}_{\text {ep }}$ & $0.685 \pm 0.548$ & $0.291 \pm 0.185$ & $0.182 \pm 0.086$ & $0.182 \pm 0.141$ & $0.142 \pm 0.053$ \\
& $\mathrm{~V}_{\mathrm{e}}$ & $0.445 \pm 0.211$ & $0.565 \pm 0.196$ & $0.598 \pm 0.172$ & $0.621 \pm 0.149$ & $0.661 \pm 0.130$ \\
\multirow{3}{*}{ Malignant } & $\mathrm{K}_{\text {trans }}$ & $1.333 \pm 0.401$ & $0.967 \pm 0.361$ & $0.885 \pm 0.378$ & $0.848 \pm 0.314$ & $0.896 \pm 0.366$ \\
& $\mathrm{~K}_{\text {ep }}$ & $2.420 \pm 0.723$ & $1.742 \pm 0.552$ & $1.546 \pm 0.559$ & $1.514 \pm 0.662$ & $1.625 \pm 0.861$ \\
& $\mathrm{~V}_{\mathrm{e}}$ & $0.584 \pm 0.147$ & $0.581 \pm 0.137$ & $0.601 \pm 0.150$ & $0.606 \pm 0.169$ & $0.590 \pm 0.182$ \\
\hline
\end{tabular}

$\mathrm{K}_{\text {trans }}$, volume transfer constant; $\mathrm{K}_{\mathrm{ep}}$, rate constant; $\mathrm{V}_{\mathrm{e}}$, extravascular extracellular volume fraction.

Table II. Statistical significance of the three indicator values acquired at all phases in the differentiation of benign and malignant tumors (P-value).

\begin{tabular}{lrrrrr}
\hline & \multicolumn{5}{c}{ Phase } \\
\cline { 2 - 6 } Indicators & \multicolumn{1}{c}{10} & \multicolumn{1}{c}{20} & \multicolumn{1}{c}{30} & \multicolumn{1}{c}{40} & \multicolumn{1}{c}{50} \\
\hline $\mathrm{K}_{\text {trans }}$ & $<0.001$ & $<0.001$ & $<0.001$ & $<0.001$ & $<0.001$ \\
$\mathrm{~K}_{\text {ep }}$ & $<0.001$ & $<0.001$ & $<0.001$ & $<0.001$ & $<0.001$ \\
$\mathrm{~V}_{\mathrm{e}}$ & 0.015 & 0.768 & 0.950 & 0.776 & 0.201 \\
\hline
\end{tabular}

$\mathrm{K}_{\text {trans }}$, volume transfer constant; $\mathrm{K}_{\mathrm{ep}}$, rate constant; $\mathrm{V}_{\mathrm{e}}$, extravascular extracellular volume fraction.

Analysis of optimal scan window. Table III lists the statistical significance of differences of the three quantitative parameter values between different scan phases. For $\mathrm{K}_{\text {trans }}$ and $\mathrm{K}_{\mathrm{ep}}$ values, differences between the values at phases 30 and 50 as well as those between phases 30 and 40 were not statistically significant in the malignant tumor group ( $\mathrm{P}>0.05)$. In the benign tumor group, changes in $\mathrm{K}_{\text {trans }}$ and $\mathrm{K}_{\mathrm{ep}}$ values between phase 40 and phase 50 were not statistically significant $(\mathrm{P}>0.05)$. Differences of $\mathrm{V}_{\mathrm{e}}$ value between various phases in the malignant group were not found to be statistically significant $(\mathrm{P}>0.05)$. Differences of $\mathrm{V}_{\mathrm{e}}$ value between phases 30 and 40 in the benign tumor group were also not statistically significant $(\mathrm{P}>0.05)$. The $\mathrm{K}_{\text {trans }}, \mathrm{K}_{\mathrm{ep}}$ and $\mathrm{V}_{\mathrm{e}}$ value analysis confirmed that the optimal scanning time period was between phase 10 and phase 30 after contrast agent injection.

\section{Discussion}

DCE-MRI is capable of detecting changes of the microvascular structures in tissue, and is particularly useful for the targeted imaging of tumor angiogenesis. Though the analysis of this imaging modality is rather simple, the three parameters $\mathrm{K}_{\text {trans }}, \mathrm{K}_{\mathrm{ep}}$ and $\mathrm{V}_{\mathrm{e}}$ are able to evaluate the contrast agent diffusion dynamics and hemodynamics in tissues accurately and improve the diagnostic efficacy for breast cancer patients (9). They are effective indicators of the physiological state based on dual-compartmental pharmacokinetic model analysis, and therefore are widely adopted in tumor imaging study (10). El Khouli et al (11) and some other studies suggest that $\mathrm{K}_{\text {trans }}$ and $\mathrm{K}_{\mathrm{ep}}$ values are statistically significant in the diagnosis of benign and malignant breast tumors $(12,13)$. Li et al (14) showed that the $\mathrm{K}_{\text {trans }}$ value was distinctly higher in malignant breast lesions than in benign breast lesions. Amarnath et al (15) concluded that $\mathrm{K}_{\text {trans }}$ used in breast DCE-MRI is a reliable quantitative parameter for identifying benign and malignant lesions. In another study, Li et al (16) demonstrated that $\mathrm{K}_{\text {trans }}$ and $\mathrm{K}_{\mathrm{ep}}$ values were reduced in benign breast lesions compared with maliganancies. The present study shows similar results, indicating that the $\mathrm{K}_{\text {trans }}$ and $\mathrm{K}_{\mathrm{ep}}$ values of malignant mammary gland lesions were significantly higher than those of benign lesions, and that the difference was statistically significant.

The prostate quantitative parameter analysis conducted by Ocak et al (17) showed that the $\mathrm{K}_{\text {trans }}$ and $\mathrm{K}_{\mathrm{ep}}$ values of tumor tissue are markedly higher than those in the normal peripheral zone, while $V_{e}$ values indicated no significant difference between the two lesions. The diagnostic efficacy of changes in $\mathrm{V}_{\mathrm{e}}$ values in benign and malignant tumors remains debatable. The study by Koo et al (10) indicated that the values of $\mathrm{V}_{\mathrm{e}}$ would decrease as malignant breast tumor progresses to a higher stage. The present study shows that $\mathrm{V}_{\mathrm{e}}$ values have no significant difference between benign and malignant lesions which is consistent with the study by Ocak et al (17). The authors of the present study attempted to observe the trend in changes by analyzing DCE images at multi-phases, and to explore the significance of the trend in the identification of lesions. The multiple phase analysis showed the that $\mathrm{V}_{\mathrm{e}}$ value increased in benign lesions as the scan phase increased, while it exhibited no obvious changes in malignant tissue. The slope of $\mathrm{V}_{\mathrm{e}}$ value change may be a valuable diagnosis surrogate in the differentiation of benign and malignant lesions. Our hypothesis for the interpretation of this phenomenon is that the endothelial structure is more mature in benign lesions than in malignant lesions; therefore, the filling of contrast agent into the interstitial structure changes with time in benign lesions, but remains steady in malignant lesions.

Few studies have been conducted for exploration of the optimized scanning time window of breast DCE-MRI, due to the large variety of scanning equipment, contrast agents and injection speed. In the present study, the scanning time window, was increased and the indicative parameters at multiple scan 
Table III. Statistical significance of differences between the three indicator values acquired at any two phases (P-value).

\begin{tabular}{|c|c|c|c|c|c|c|}
\hline \multirow[b]{2}{*}{ Phases } & \multicolumn{2}{|c|}{$\mathrm{K}_{\text {trans }}$} & \multicolumn{2}{|c|}{$\mathrm{K}_{\mathrm{ep}}$} & \multicolumn{2}{|c|}{$\mathrm{V}_{\mathrm{e}}$} \\
\hline & Benign & Malignant & Benign & Malignant & Benign & Malignant \\
\hline 10 vs. 20 & 0.009 & $<0.001$ & 0.003 & $<0.001$ & $<0.001$ & 0.801 \\
\hline 10 vs. 30 & 0.005 & $<0.001$ & 0.004 & $<0.001$ & $<0.001$ & 0.222 \\
\hline 10 vs. 40 & 0.003 & $<0.001$ & 0.002 & $<0.001$ & 0.001 & 0.154 \\
\hline 10 vs. 50 & 0.003 & $<0.001$ & 0.002 & $<0.001$ & $<0.001$ & 0.753 \\
\hline 20 vs. 30 & 0.002 & $<0.001$ & 0.017 & 0.001 & 0.044 & 0.035 \\
\hline 20 vs. 40 & 0.002 & 0.005 & 0.020 & 0.004 & 0.098 & 0.064 \\
\hline 20 vs. 50 & 0.002 & 0.213 & 0.003 & 0.281 & 0.008 & 0.553 \\
\hline 30 vs. 40 & 0.016 & 0.376 & 0.995 & 0.724 & 0.415 & 0.630 \\
\hline 30 vs. 50 & 0.020 & 0.847 & 0.005 & 0.535 & 0.022 & 0.352 \\
\hline 40 vs. 50 & 0.145 & 0.074 & 0.172 & 0.042 & 0.041 & 0.076 \\
\hline
\end{tabular}

$\mathrm{K}_{\text {trans }}$, volume transfer constant; $\mathrm{K}_{\mathrm{ep}}$, rate constant; $\mathrm{V}_{\mathrm{e}}$, extravascular extracellular volume fraction.
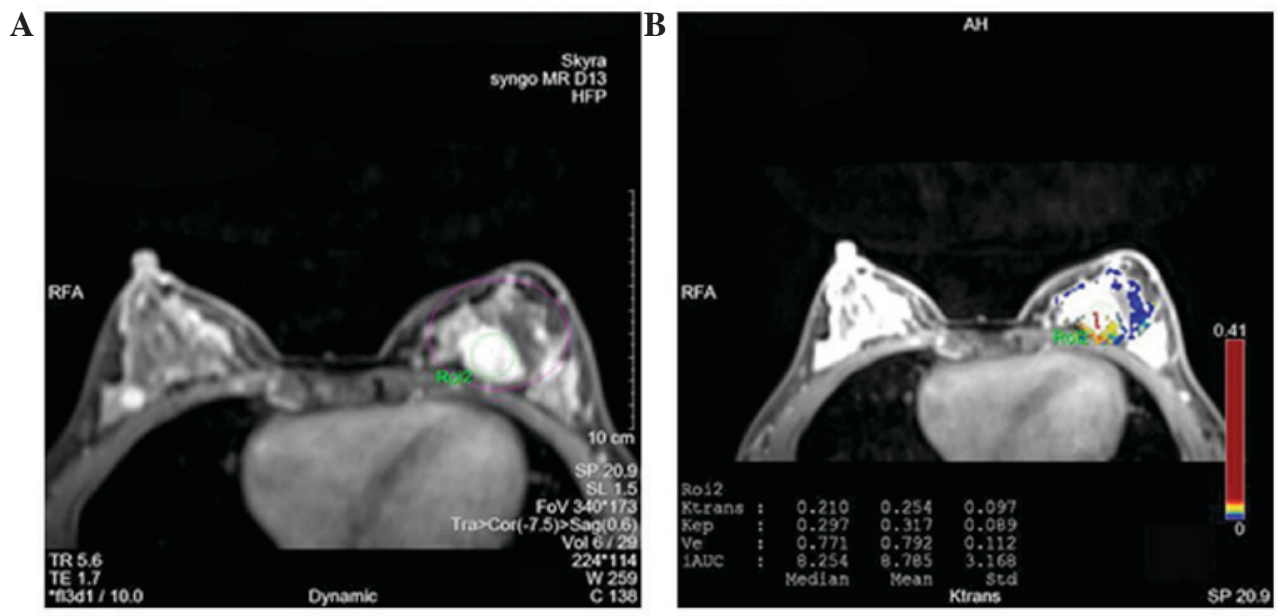

Figure 3. DCE images of a 23-year-old female with left breast fibro-adenoma. (A) Early phase DCE image: A late stage circular uniform region with enhanced signal is shown deeply under the left breast. (B) DCE pseudo-color map image: The lump region shows elevated diffusion pseudo-color yellow and red. Volume transfer constant, $0.210 \mathrm{~min}^{-1}$; rate constant, $0.297 \mathrm{~min}^{-1}$; extravascular extracellular volume fraction, 0.771; integrated area under the curve, 8.254 . DCE, dynamic contrast enhancement.
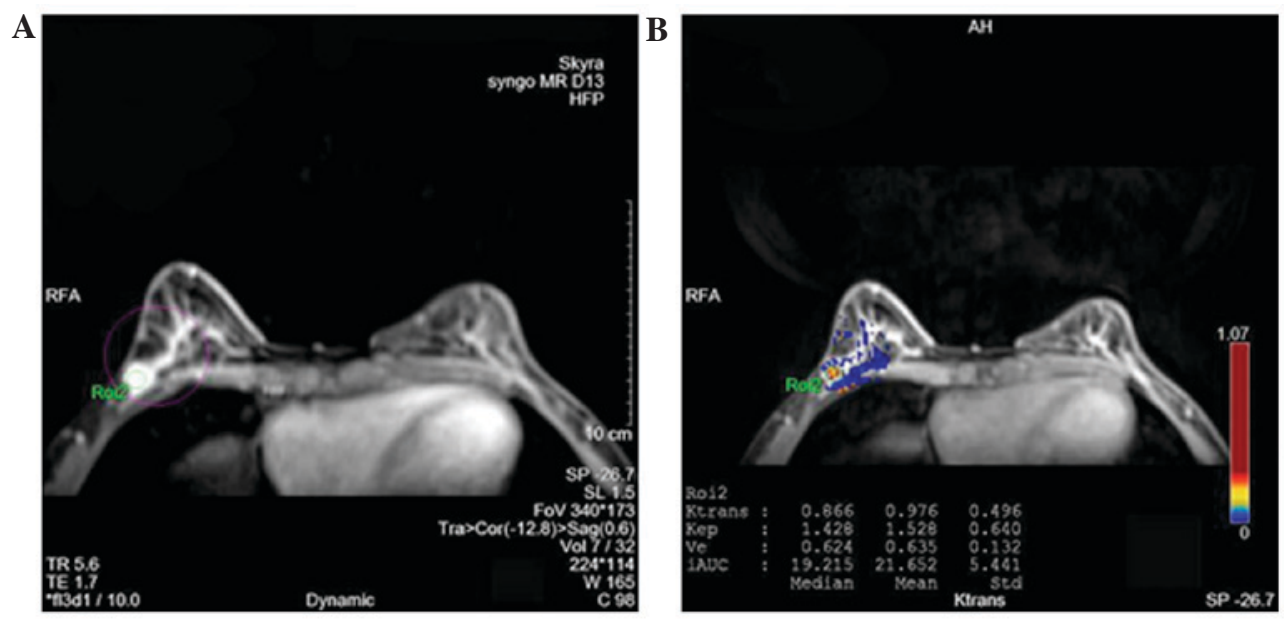

Figure 4. DCE images of a 56-year-old female patient with infiltrating ductal carcinoma in the right breast. (A) Early phase DCE image: Early abnormal region with enhanced signal is shown, (B) DCE pseudo-color map: Lump area shows low diffusion pseudo-color of blue and yellow. Volume transfer constant, $0.866 \mathrm{~min}^{-1}$; rate constant, $1.428 \mathrm{~min}^{-1}$; extravascular extracellular volume fraction, 0.624 ; integrated area under the curve, 19.215. DCE, dynamic contrast enhancement. 
phases were analyzed, and a conclusion regarding the optimized scanning window following contrast injection was reached. The results demonstrated that the optimized scanning window is between phase 10 and phase 30, namely between 5 and 15 min after contrast injection, and the single scan time is $30 \mathrm{sec}$. In order to reduce scan time and achieve higher equipment usage efficiency, the best practice is scanning for 5 min following the contrast injection. In order to achieve better diagnostic specificity, the semi-quantitative indicator, time-intensity curve and the $\mathrm{V}_{\mathrm{e}}$ value change trend should be considered by scanning another $15 \mathrm{~min}$ after contrast injection.

In conclusion, this study shows that $\mathrm{K}_{\text {trans }}$ and $\mathrm{K}_{\mathrm{ep}}$ values achieved from 3.0 T DCE-MRI have statistically significant value in the identification of benign and malignant breast lesions, and that the trend in $\mathrm{V}_{\mathrm{e}}$ value changes can also be used as a supplemental reference for specifying benign and malignant breast lesions. The optimized scanning window following contrast agent injection was determined based on the above analysis. Considering the great variety of scanning equipment, contrast agents and injection speeds, and the widely spaced time data points analyzed in this study, a large cohort of patients, more precise time data point analysis and more imaging sequence parameter comparison are required to provide stronger theoretical support for optimized breast DCE-MRI scanning time in further research.

\section{Acknowledgements}

This research was funded by the Project of medical and health technology development program in Shandong province (grant no. 2013WS0124).

\section{References}

1. Aberle DR, Chiles C, Gatsonis C, Hillman BJ, Johnson CD, McClennan BL, Mitchell DG, Pisano ED, Schnall MD and Sorensen AG; American College of Radiology Imaging Network: Imaging and cancer: Research strategy of the American College of Radiology Imaging Network. Radiology 235: 741-751, 2005.

2. Leung JW: Screening mammography reduces morbidity of breast cancer treatment. AJR Am J Roentgenol 184: 1508-1509, 2005

3. Peters NH, Borel Rinkes IH, Zuithoff NP, Mali WP, Moons KG and Peeters PH: Meta-analysis of MR imaging in the diagnosis of breast lesions. Radiology 246: 116-124, 2008.
4. Jones EF, Sinha SP, Newitt DC, Klifa C, Kornak J, Park CC and Hylton NM: MRI enhancement in stromal tissue surrounding breast tumors: Association with recurrence free survival following neoadjuvant chemotherapy. PloS One 8: e61969, 2013.

5. Li X, Arlinghaus LR, Ayers GD, Chakravarthy AB, Abramson RG Abramson VG, Atuegwu N, Farley J, Mayer IA, Kelley MC, et al: DCE-MRI analysis methods for predicting the response of breast cancer to neoadjuvant chemotherapy: Pilot study findings. Magn Reson Med 71: 1592-1602, 2104.

6. Baek HM, Chen JH, Nie K, Yu HJ, Bahri S, Mehta RS, Nalcioglu $\mathrm{O}$ and Su MY: Predicting pathologic response to neoadjuvant chemotherapy in breast cancer by using MR imaging and quantitative ${ }^{1} \mathrm{H}$ MR spectroscopy. Radiology 251 : 653-662, 2009.

7. Magometschnigg HF, Helbich T, Brader P, Abeyakoon O, Baltzer P, Füger B, Wengert G, Polanec S, Bickel H and Pinker K: Molecular imaging for the characterization of breast tumors. Expert Rev Anticancer Ther 14: 711-722, 2014.

8. Rahbar $\mathrm{H}$ and Partridge SC: Multiparametric MR Imaging of Breast Cancer. Magn Reson Imaging Clin N Am 24: 223-238, 2016.

9. Petralia G, Bonello L, Priolo F, Summers P and Bellomi M: Breast MR with special focus on DW-MRI and DCE-MRI. Cancer Imaging 11: 76-90, 2011.

10. Koo HR, Cho N, Song IC, Kim H, Chang JM, Yi A, Yun BL and Moon WK: Correlation of perfusion parameters on dynamic contrast-enhanced MRI with prognostic factors and subtypes of breast cancers. J Magn Reson Imaging 36: 145-151, 2012.

11. El Khouli RH, Macura KJ, Kamel IR, Jacobs MA and Bluemke DA: 3-T dynamic contrast-enhanced MRI of the breast: Pharmacokinetic parameters versus conventional kinetic curve analysis. AJR Am J Roentgenol 197: 1498-1505, 2011.

12. Ma ZS, Wang DW, Sun XB, Shi H, Pang T, Dong GQ and Zhang CQ: Quantitative analysis of 3-Tesla magnetic resonance imaging in the differential diagnosis of breast lesions. Exp Ther Med 9: 913-918, 2015

13. Yim H, Kang DK, Jung YS, Jeon GS and Kim TH: Analysis of kinetic curve and model-based perfusion parameters on dynamic contrast enhanced MRI in breast cancer patients: Correlations with dominant stroma type. Magn Reson Imaging 34: 60-65, 2016.

14. Li J, Yu Y, Zhang Y, Bao S, Wu C, Wang X, Li J, Zhang X and $\mathrm{Hu}$ J: A clinically feasible method to estimate pharmacokinetic parameters in breast cancer. Med Phys 36: 3786-3794, 2009.

15. Amarnath J, Sangeeta T and Mehta SB: Role of quantitative pharmacokinetic parameter (transfer constant: K(trans)) in the characterization of breast lesions on MRI. Indian J Radiol Imaging 23: 19-25, 2013.

16. Li L, Wang K, Sun X, Wang K, Sun Y, Zhang G and Shen B: Parameters of dynamic contrast-enhanced MRI as imaging markers for angiogenesis and proliferation in human breast cancer. Med Sci Monit 21: 376-382, 2015.

17. Ocak I, Bernardo M, Metzger G, Barrett T, Pinto P, Albert PS and Choyke PL: Dynamic contrast-enhanced MRI of prostate cancer at $3 \mathrm{~T}$ : A study of pharmacokinetic parameters. AJR Am J Roentgenol 189: 849, 2007. 